\title{
Decreased mesenteric blood flow supplying retrosternal esophageal ileocoloplastic grafts during positive-pressure breathing
}

\begin{abstract}
Esophageal replacement after esophagogastric injury caused by ingestion of lye may require the interposition of a retrosternal ileocolic graft. In this new anatomic situation, the mesenteric circulation supplying the graft is subjected to the intrathoracic pressure surrounding the graft. Thus, mesenteric blood flow supplying the graft may be impaired when intrathoracic pressure is increased during mechanical ventilation. This study was designed to evaluate the effect of increasing intrathoracic pressure by application of a positive end-expiratory pressure on mesenteric blood flow supplying esophageal ileocolic grafts. Eight cases were studied in the immediate postoperative period. Miniaturized implantable Doppler microprobes were sutured to the single artery supplying the graft and connected to an $8 \mathrm{MHz}$ pulsed Doppler flowmeter. Two sets of measurements were successively performed with zero end-expiratory pressure ventilation and after application of a $15 \mathrm{~cm}$ water positive end-expiratory pressure. Positive end-expiratory pressure induces mean arterial pressure $(-12 \%)$; $p<0.05)$ and cardiac output $(-17 \% ; p<0.05)$ decrease. Mesenteric blood flow also decreases $(-38 \%$; $p<0.05)$ as did the mesenteric blood flow/cardiac output ratio, suggesting a potential mesenteric vasoconstriction assessed by mesenteric vascular resistance increase and mesenteric diastolic blood flow velocity decrease. These results suggest that, in the particular anatomic situation of the graft, increased intrathoracic pressure induces mesenteric blood flow decrease in relation to systemic hemodynamic alterations associated with perivisceral pressure increase. This change may be deleterious to graft perfusion. (J ThORAC CaRdiovasC SURG 1994;107:68-73)
\end{abstract}

L. Jacob, MD, S. Boudaoud, MD, O. Rabary, MD, D. Payen, MD, PhD, E. Sarfati, MD,

D. Gossot, MD, E. Rolland, MD, B. Eurin, MD, and M. Celerier, MD, Paris France

E sophagectomy for neoplastic lesion or after esophageal injury caused by ingestion of lye may necessitate esophageal replacement. This esophageal reconstruction may be achieved by interposition of a colonic graft. ${ }^{1}$ Out-

From the Service d'Anesthésiologie-Réanimation Chirurgicale, Hôpital Universitaire Saint-Louis, Paris, France, and from the Service d'Anesthésiologie-Reanimation Chirurgicale, Hôpital Universitaire Lariboisière, Paris, France.

Supported by Faculty Institutional grant program of Faculté de Médecine Lariboisière-Saint-Louis, Université Paris VII, Fédération Française de Cardiologie and Institut National de la Santé et de la Recherche Médicale (INSERM), contract No. 86-3-37-E.

Received for publication Jan. 13, 1993.

Accepted for publication May 24, 1993.

Address for reprints: Laurent Jacob, MD, Service d'AnesthésiologieRéanimation Chirurgicale, Hôpital Saint-Louis, 1 Ave. Claude Vellefaux, 75010 Paris, France.

Copyright $\odot 1994$ by Mosby-Year Book, Inc.

$0022-5223 / 94 \$ 1.00+.10 \quad \mathbf{1 2} / \mathbf{1} / \mathbf{4 9 6 1 0}$ come after this procedure may be compromised by cervical anastomosis leakage or stenosis, the prevalence of which ranges from $33 \%$ to $56 \%{ }^{2-5}$ These complications are often related to regional hypoperfusion and ischemia produced by systemic hemodynamic alteration ${ }^{6}$ or impairment of local blood flow. ${ }^{3}$ The graft blood supply is achieved by vessels extending from the abdominal to the thoracic compartment. Venous drainage of the graft continued in the portal system. These esophagoplastic grafts therefore represent a model in which a part of the splanchnic circulation is brought in the thorax and subjected to the intrathoracic pressure surrounding the graft. Because esophageal replacement is performed while the patient is under general anesthesia and mechanical ventilation is often required during the postoperative period, local mesenteric blood flow (MBF) supplying the graft may be impaired when intrathoracic pressure is increased during mechanical ventilation.

This study was conducted to evaluate with implantable 
Doppler microprobes ${ }^{7,8}$ the consequences of increasing intrathoracic pressure by application of a $15 \mathrm{~cm} \mathrm{H}_{2} \mathrm{O}$ positive end-expiratory pressure (PEEP) on MBF supplying esophageal ileocolic grafts.

\section{Patients and methods}

Patients. Eight patients, $29 \pm 4$ years of age (mean \pm standard deviation), were included in the study after informed consent and local ethical committee approval were obtained. Patients had no history of cardiovascular or respiratory disease. Esophageal reconstruction was scheduled several months after severe caustic burns of the esophagus and stomach. In brief the operative method used has been previously described ${ }^{1,9,10}$ and consisted of a blunt retrosternal dissection. The mobilized ileocolic graft was brought through this newly created anterior mediastinal tunnel and anastomosed to the cervical esophagus in an isoperistaltic orientation. Vascular supply of the graft was achieved by the right upper colic artery, a branch of the superior mesenteric artery, and venous drainage continued into the portal system.

Systemic hemodynamic parameters. The following parameters were measured or calculated: heart rate (HR), as seen with electrocardiogram; mean systemic arterial pressure (MAP) with a radial catheter; and right atrial pressure, pulmonary arterial pressure, and pulmonary artery occlusion pressure with a triple lumen catheter. All end-expiratory pressures were measured with Hewlett-Packard quartz transducers (Hewlett-Packard, Palo Alto, Calif.) carefully zeroed at the midaxillary level. Cardiac output $(\mathrm{CO})$ values were obtained by averaging three successive thermodilution determinations (CO computer 9510 A; Edwards Laboratories, Santa Ana, Calif.). Cardiac index (CI) and systemic vascular resistance index was calculated as (MAP - RAP)/CI in mm $\mathrm{Hg} \cdot \mathrm{L}^{-1} \cdot \mathrm{min} \cdot \mathrm{m}^{2}=\mathrm{IU}$, where $\mathrm{RAP}$ is right atrial pressure.

Simultaneous systematic and mixed venous blood samples were obtained for blood gas analysis (ABL 30 Radiometer A/S, Copenhagen, Denmark). Arterial and mixed central venous oxygen contents were derived from arterial and mixed venous, oxygen tension and oxygen saturation. Arteriovenous difference in oxygen contents was calculated from these values: $\mathrm{CAO}_{2}-\mathrm{C} \bar{v}_{2}$ (arterial and mixed central venous oxygen contents, respectively). Systemic oxygen delivery was calculated as $\mathrm{CI} \cdot \mathrm{CAO}_{2} \cdot 10\left(\mathrm{ml} \cdot \mathrm{min}^{-1} \cdot \mathrm{m}^{-2}\right)$, where $\mathrm{CI}$ is cardiac index.

Intrathoracic pressure. A fluid-filled catheter linked to a pressure transducer placed at the midaxillary level was positioned in the graft at the carina level to measure intragraft pressure.
Mesenteric blood'flow study. The Doppler microprobe design and implantation technique have been previously described. ${ }^{7}$ In brief, the probes were $3 \mathrm{~mm}$ in width and $4 \mathrm{~mm}$ in length. The Doppler crystal was glued to a silicone prism in such a manner as to obtain an incidence angle of 60 degrees between the ultrasonic beam and the vessel axis. Before implantation, the linearity of each probe response for flow velocities ranging from 5 to $80 \mathrm{~cm} \cdot \mathrm{sec}^{-1}$ was verified. ${ }^{7}$ At the end of the surgical procedure, a single-use $8 \mathrm{MHz}$ pulsed Doppler microprobe was sutured to the adventitia of the right upper colic artery near its origin. To facilitate implantation and proper alignment of the probe along the vessel axis, we cut the base of the silicone prism in the shape of a concave gauge adapted to vessel curvilinearity. Four 7-0 sutures, carefully passed through the arterial adventitia and through the silicone prism, enabled close contact between the probe and the vessel wall with proper alignment in relation to vessel axis.

The probe was connected to the Doppler flowmeter with leads exiting from the abdomen through the skin. The zero-crossing pulsed Doppler blood flowmeter used in this study has previously been described and validated. ${ }^{7-11}$ The main characteristic of this apparatus is the range-gated time system of reception, which enables selection of sample volume size and movement across the vessel lumen. The diameter was determined according to the echographic equation: $\mathrm{C} / 2 \cdot(\mathrm{t} 2-\mathrm{t} 1) \cdot \cos 60$ degrees, where $\mathrm{C}$ is the ultrasound speed in biologic tissues, $\mathrm{t} 2-\mathrm{t} 1$ is the difference between distal and proximal reception time corresponding to the vessel walls, and 60 degrees is the incidence angle of the ultrasonic beam. ${ }^{11}$ Mean (Vm) and phasic cross-sectional blood flow velocities can be measured. Mean mesenteric blood flow (in $\mathrm{ml} \cdot \min ^{-1}$ ) was calculated according to the formula: $\pi$ $\mathrm{D}^{2} / 4 \cdot \mathrm{Vm}$, where $\mathrm{D}$ is arterial diameter. Because arterial diameter was assumed to be constant all along the cardiac cycle, only maximum systolic and end-diastolic blood flow velocities were recorded. Because the true back pressure of the flow was impossible to measure, we calculated the index of local mesenteric vascular resistance as the direct ratio of $\mathrm{MAP} /$ mean $\mathrm{MBF}$ (in $\mathrm{mm} \mathrm{Hg} \cdot \mathrm{L}^{-1}$ - min). Local mesenteric oxygen delivery was calculated as follows: $\mathrm{MBF} \cdot \mathrm{CAO}_{2}$ (in $\mathrm{ml} / \mathrm{min}$ ), where $\mathrm{CAO}_{2}$ is arterial oxygen content. The probes were removed 3 days later by gentle traction with no adverse effect.

Protocol. Systemic and mesenteric hemodynamic parameters were measured 3 hours after the operation when hemodynamic conditions were stable and after checking clinical and laboratory parameters (central temperature was $36.3^{\circ} \pm 0.7^{\circ} \mathrm{C}$ and plasma hemoglobin concentration was $11.1 \pm 1.6 \mathrm{gm} / 100 \mathrm{ml}$ ). During the postoperative and the protocol periods, fentanyl was con- 
Table I. Mean systemic hemodynamic parameters during $Z E E P$ and PEEP ventilation $(n=8)$

\begin{tabular}{lcc} 
& ZEEP & PEEP \\
\hline $\mathrm{MAP}(\mathrm{mm} \mathrm{Hg})$ & $94 \pm 12$ & $83 \pm 12^{*}$ \\
$\mathrm{HR}($ beats/min$)$ & $92 \pm 20$ & $92 \pm 17$ \\
$\mathrm{CO}(\mathrm{L} / \mathrm{min})$ & $7.2 \pm 1.7$ & $6 \pm 1.6^{*}$ \\
$\mathrm{RAP}(\mathrm{mm} \mathrm{Hg})$ & $7.5 \pm 2.1$ & $11.3 \pm 2.9^{*}$ \\
$\mathrm{SVRI}\left(\mathrm{mm} \mathrm{Hg} \cdot \mathrm{L} \cdot \mathrm{min} \cdot \mathrm{m}^{2}\right.$ & $19.5 \pm 3.1$ & $19.8 \pm 4.6^{*}$ \\
$\mathrm{PaO}_{2}(\mathrm{~mm} \mathrm{Hg})$ & $174 \pm 57$ & $196 \pm 49^{*}$ \\
$\mathrm{SaO}_{2}(\%)$ & $99.4 \pm 0.51$ & $99.5 \pm 0.42$ \\
$\mathrm{PaCO}_{2}(\mathrm{~mm} \mathrm{Hg})$ & $31 \pm 8.7$ & $31.6 \pm 6.2$ \\
$\mathrm{AV} \mathrm{DO}_{2}(\mathrm{ml} / 100 \mathrm{ml})$ & $3.02 \pm 0.8$ & $4.01 \pm 1.5^{*}$ \\
$\mathrm{DO}_{2}(\mathrm{ml} / \mathrm{min})$ & $694 \pm 185$ & $578 \pm 178^{*}$
\end{tabular}

Results are mean \pm standard deviation. $H R$, Heart rate; $R A P$, right atrial pressure; $S V R I$, systemic vascular resistance index; $\mathrm{PaO}_{2}$, arterial oxygen tension; $\mathrm{PaCO}_{2}$, arterial carbon dioxide tension; $\mathrm{SaO}_{2}$, arterial oxygen saturation $\mathrm{AVDO}$, arteriovenous difference in oxygen contents; $\mathrm{DO}_{2}$, systemic oxygen delivery. ${ }^{*} p<0.05$ versus ZEEP.

tinuously infused $\left(300 \mu \mathrm{g} \cdot \mathrm{hr}^{-1}\right)$. Lungs were mechanically ventilated via an endotracheal tube with a volume cycled ventilator (CPU 1; Ohmeda, Maurepas, France) with an inspired oxygen fraction of 0.40 . Minute ventilation and tidal volume were adjusted to maintain arterial carbon dioxide tension between 30 and $40 \mathrm{~mm} \mathrm{Hg}$.

Two sets of measurements were successively performed after 10-minute periods of equilibration during zero endexpiratory pressure ventilation (ZEEP) and during application of a $15 \mathrm{~cm}$ water PEEP.

Statistical analysis. Results are expressed as mean \pm standard deviation. Comparison between measurements was achieved by a nonparametric Wilcoxon paired test; $p<0.05$ was considered significant.

\section{Results}

Implantation and removal of Doppler microprobes were not associated with any adverse effect on the graft perfusion or outcome.

Systematic hemodynamic parameters are summarized in Table I. PEEP induced a $17 \%$ CO decrease $(p<0.05)$ and a 12\% MAP reduction $(p<0.05)$. Oxygen delivery decreased from $694 \pm 185$ to $578 \pm 178 \mathrm{ml} \cdot \mathrm{min}^{-1}$ $\cdot \mathrm{m}^{-2}(p<0.05)$. Arteriovenous difference in oxygen contents increased from $3 \pm 0.8 \mathrm{ml} / 100 \mathrm{ml}$ to $4 \pm 1.5$ $\mathrm{ml} / 100 \mathrm{ml}(p<0.05)$.

Mean end-expiratory intragraft pressure increased from $6.5 \pm 0.9 \mathrm{~mm} \mathrm{Hg}$ to $12.7 \pm 1.1 \mathrm{~mm} \mathrm{Hg}$.

Regional mesenteric hemodynamic parameters are summarized in Table II. Mean intraluminal colic artery diameter was $3.1 \pm 1.2 \mathrm{~mm}$ (range 1.9 to $4.4 \mathrm{~mm}$ ). PEEP ventilation induced a $38 \%$ decrease in $\operatorname{MBF}(p<0.05)$ and a $53 \%$ increase in mesenteric vascular resistance index $(p<0.05)$. The MBF/CO ratio decreased from
Table II. Mean mesenteric hemodynamic parameters values during $Z E E P$ and PEEP ventilation $(n=8)$

\begin{tabular}{lcc}
\hline & ZEEP & \multicolumn{1}{c}{ PEEP } \\
\hline $\mathrm{MBF}(\mathrm{ml} / \mathrm{min})$ & $43 \pm 39$ & $24 \pm 20^{*}$ \\
$\mathrm{MVR}(\mathrm{mm} \mathrm{Hg} \cdot \mathrm{L} \cdot \mathrm{min})$ & $44.1 \pm 39$ & $60 \pm 54^{*}$ \\
$\mathrm{MBF} / \mathrm{CO}(\%)$ & $0.59 \pm 0.6$ & $0.41 \pm 0.4^{*}$ \\
$\mathrm{Vs}(\mathrm{cm} / \mathrm{sec})$ & $29.3 \pm 7.1$ & $24.5 \pm 8.5^{*}$ \\
$\mathrm{Vd}(\mathrm{cm} / \mathrm{sec})$ & $2 \pm 2$ & $-0.8 \pm 1.4^{*}$ \\
$\mathrm{DmO}(\mathrm{ml} / \mathrm{min})$ & $6.4 \pm 6$ & $3.6 \pm 2.8^{*}$ \\
$\mathrm{Pg}(\mathrm{mm} \mathrm{Hg})$ & $6.5 \pm 2.5$ & $12.7 \pm 3.1^{*}$ \\
\hline
\end{tabular}

Results are mean \pm standard deviation. $M V R$, Mesenteric vascular resistance; $V_{s}$, systolic mesenteric blood flow velocity; $V d$, diastolic mesenteric blood flow velocity; $\mathrm{DmO}_{2}$, mesenteric oxygen delivery; $\mathrm{Pg}$, intragraft pressure.

${ }^{*} \mathrm{p}<0.05$ versus ZEEP.

$0.59 \% \pm 0.6 \%$ to $0.41 \% \pm 0.4 \%(p<0.05)$. Mean systolic $\quad\left(29.3 \pm 7.1\right.$ versus $24.5 \pm 8.5 \mathrm{~cm} \cdot \mathrm{sec}^{-1}$; $(p<0.05)$ and diastolic $(2 \pm 2$ versus $-0.8 \pm 1.4$ $\mathrm{cm} \cdot \sec ^{-1} ;(p<0.05)$ blood flow velocities decreased during PEEP. Local oxygen delivery decreases from $6.4 \pm 6$ to $3.6 \pm 2.8 \mathrm{ml} \cdot \mathrm{min}^{-1}(p<0.05)$. Fig. 1 shows a typical tracing in one patient. In this patient, the diastolic mesenteric blood flow velocity, which was close to zero during ZEEP, decreased to a negative value during PEEP.

\section{Discussion}

Clinical outcome after esophageal replacement by interposition of an ileocolic graft might be compromised by local complications such as stenosis or anastomosis leakage. 2,3,6 Because local ischemia seems to be involved, ${ }^{6}$ monitoring of local blood flow may be of interest to evaluate which factors may induce such a local ischemic condition. Because esophageal replacement may require mechanical ventilation to prevent or treat respiratory failure in the postoperative period, this ventilatory support may have some adverse effects on graft vascularization. According to our findings with Doppler technology, ${ }^{7,8}$ we report the effect of increased intrathoracic pressure with PEEP ventilation on MBF after esophageal replacement by an ileocolic graft. In the part of the mesenteric circulation that is brought into the thorax, PEEP induces an important MBF decrease whose magnitude was larger than what would be observed if the gut were in the abdomen.

Perioperative measurements of human mesenteric blood flow were not possible until the development of implantable flow probes. The technique used in this study has been described previously ${ }^{7}$ and was used to measure aorta-coronary bypass flow, ${ }^{7}$ hepatic blood flow after orthoptic liver transplantation, ${ }^{8}$ mesenteric blood flow, 9,10 or renal blood flow after kidney transplanta- 
tion. ${ }^{12}$ The technique used has the following advantages: a small and portable system allowing bedside measurements, a simple and rapid measurement procedure, and continuous monitoring if needed. The accuracy of Doppler flow measurement technique has been previously described in experimental ${ }^{11}$ and clinical studies. ${ }^{7-10,12}$ Precise blood flow measurements with this technique require exact determination of vessel internal diameter. With the same equipment, Levenson and associates ${ }^{11}$ demonstrated the accuracy of pulsed Doppler flow diameter measurements, comparing Doppler flow diameter determination with actual diameter of calibrated tubes with the use of an in vitro model. Moreover, in this study, comparisons were made between repetitive measurements in the same patient, limiting the impact of a systematic error.

Because intraoperative mesenteric flow measurements cannot be interpreted because of the vascular impact of surgical manipulations, the systemic and mesenteric hemodynamic measurements were collected immediately after operation while the patient was under continuous sedation with fentanyl infusion. For the same reasons, intraoperative control measurements of PEEP-induced $\mathrm{MBF}$ variation could not be performed before the ileocolic graft was brought into the thorax.

Compared with ZEEP, PEEP induced standard systemic hemodynamic changes, such as MAP and $\mathrm{CO}$ decrease. These hemodynamic alterations may be related to PEEP-induced impairment of venous return. ${ }^{13-16}$ Because arterial oxygen saturation was not significantly modified by PEEP in patients without pulmonary ventilation-perfusion mismatch, systemic oxygen delivery decreased during PEEP according to CO decrease. Arteriovenous difference in oxygen contents increased during PEEP, indirectly validating $\mathrm{CO}$ decrease and suggesting an increase in peripheral oxygen extraction. Arterial carbon dioxide tension was not significantly modified during PEEP ventilation, and $\mathrm{pH}$ values remained stable $(7.4 \pm 0.05$ versus $7.41 \pm 0.05)$; any systemic acidosis interference with MBF variation during the protocol period is therefore ruled out.

In the native esophagus, where the lumina are virtual, intraluminal end-expiratory pressures are correlated to intrapleural and intrathoracic pressure. This parameter was not available in our patients. The graft lumen was filled with some liquid and air, and this may account for the PEEP observed during ZEEP. PEEP ventilation was associated with an increase of the pressure measured in the lumen of the graft. End-expiratory intragraft pressure did not reflect the actual intrathoracic pressure, but its increase with PEEP suggested that perivisceral pressure increased.

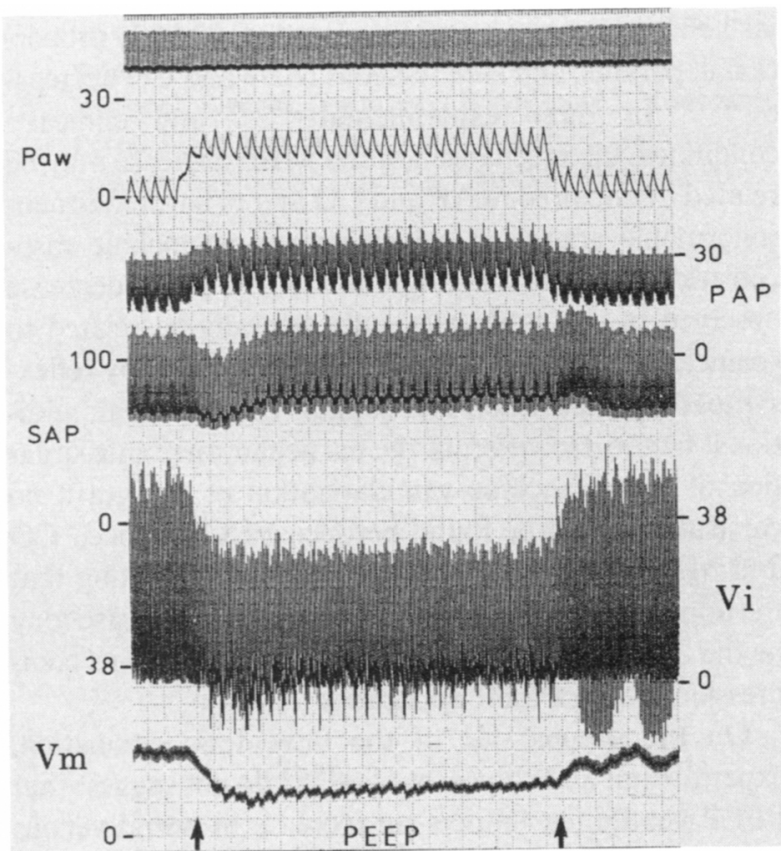

Fig. 1. Typical tracing in one patient during ZEEP and PEEP. $P a w$, Airway pressure; $P A P$, pulmonary arterial pressure; $S A P$, systemic arterial pressure; $V i, V m$, instantaneous and mean crosssectional blood flow velocities in the artery supplying the graft, respectively.

Mesenteric blood flow study showed a dispersion in MBF values, ranging from 8.9 to $122 \mathrm{ml} \cdot \mathrm{min}^{-1}$, which may be explained by arterial diameter difference. This variability in ileocolic arterial vascularization assessed by pulsed Doppler measurements is in accordance with anatomic and angiographic evidence. The wide range of MBF values may also be related to the difference between the graft weight. Despite the wide range of right upper colic artery diameter values, the PEEP-induced MBF decrease was observed in all patients (ranging from $-20 \%$ to $-60 \%$ ). No relationship could be found between arterial diameter and the magnitude of the PEEP-induced MBF decrease.

During PEEP, MBF decreased twice as much as did $\mathrm{CO}$. This fall in MBF was associated with a mesenteric vascular resistance increase and with the $\mathrm{MBF} / \mathrm{CO}$ ratio and mean diastolic blood flow velocity decrease. During PEEP, diastolic mesenteric blood flow velocity was zero in six patients and became negative in two patients, which suggests an increase in downstream vascular resistance $9,17,18$ in this part of the splanchnic circulation during PEEP application.

During PEEP, mesenteric vascular resistance increase may be related to either arteriolar vasoconstriction or to venous outflow impairment. When the splanchnic vascu- 
lar bed is in the abdomen, PEEP induces strictly proportional, parallel, and simultaneous MBF and CO decreases. ${ }^{19,20}$ In these experimental ${ }^{19,20}$ and clinical $^{21}$ conditions, the mechanism of the MBF decrease may be related to impaired MAP and $\mathrm{CO}$ and to baroreflex neurohormonal activation, which induces splanchnic vasoconstriction. Thus the magnitude of the MBF decrease observed in our study may only partially be related to splanchnic arteriolar vasoconstriction induced by reflexsympathetic activation and suggest the role of an additional factor. However, in the particular anatomic situation of the splanchnic vascularization of the graft, no correlation could be found between PEEP-induced CO and MBF variation $(r=0.4 ; p=0.39)$, suggesting that the additional factor involved in the MBF decrease may be the intrathoracic localization of the graft and its compression during PEEP application.

On the venous side of the mesenteric circulation, experimental and clinical studies ${ }^{19-21}$ do not suggest that PEEP ventilation induces an increase in portal venous resistance. In these studies, the PEEP-induced decrease in portal blood flow seems to be passive and proportional to the decrease in splanchnic blood flow. ${ }^{19}$ Moreover, intraluminal portal pressure increase during PEEP is minimal and strictly proportional to intraabdominal pressure increase. ${ }^{20}$ As a matter of fact, portal pressure does not increase if intraabdominal pressure does not increase when PEEP ventilation is applied during the operation in human beings when the abdomen is opened. ${ }^{21}$ All these results suggest that hepatic venous resistance is not modified during PEEP and does not account for the MVR increase observed in the circulation supplying intrathoracic ileocolic grafts. The magnitude of $\mathrm{MBF}$ decrease and the absence of evidence of hepatic outflow resistance involvement in MVR increase suggest that, in this particular anatomic situation, MBF decrease may be related to reflex splanchnic vasoconstriction associated with perivisceral pressure increase, which may squeeze the intrathoracic vessels either on the arteriolar or venous side of this circulation.

From a clinical point of view, if positive-pressure breathing must be maintained in the perioperative course of esophageal replacement by interposition of a colonic graft, special attention should be paid to systemic hemodynamic preservation to prevent impaired graft perfusion, which may induce local ischemia and compromise clinical outcome. ${ }^{10}$

\section{REFERENCES}

1. Sarfati E, Gossot D, Assens P, Celerier M. Management of caustic ingestion in adults. Br J Surg 1987;74:146-8.

2. Chien KY, Wang PY, Lu KS. Esophagoplasty for corrosive stricture of the esophagus: an analysis of 60 cases. Ann Surg 1974; 179:510-5.

3. Stone MM, Mahour GH, Weitzman JJ, Fonkarlrud EW, Takiff H. Oesophageal replacement with colon interposition in children. Ann Surg 1986;203:346-51.

4. Wilkins EW. Long segment colon substitution for the esophagus. Ann Surg 1980;192:722-5.

5. Potlethwait RW. Colonic interposition for esophageal substitution. Surg Gynecol Obstet 1983;156:377-83.

6. Schrock TR, Deveney CW, Dunphy JE. Factors contributing to leakage of colonic anastomosis. Ann Surg 1973; 177:513-8.

7. Payen D, Bousseau D, Laborde F, et al. Comparison of perioperative and postoperative phasic blood flow in aortocoronary venous bypass grafts by means of pulsed Doppler echocardiography with implantable microprobes. Circulation 1986;74(Suppl):III61-7.

8. Payen D, Fratacci MD, Dupuy P, et al. Portal and hepatic arterial blood flow measurements of human transplanted liver by implanted Doppler probes: interest for early complications and nutrition. Surgery 1990;107:417-27.

9. Jacob L, Boudaoud S, Payen D, et al. Isoflurane, and not halothane, increases mesenteric blood flow supplying esophageal ileocoloplasty. Anesthesiology 1991;74:699704.

10. Jacob L, Rabary O, Boudaoud S, et al. Interest of perioperative pulsed Doppler flowmetry in predicting postoperative local ischemic complication after esophageal ileocoloplasties. J THORAC Cardiovasc Surg 1992;104:38590.

11. Levenson JA, Perronneau PP, Simon ACh, Safar ME. Pulsed Doppler: determination of diameter, blood flow velocity and volumic flow of brachial artery in man. Cardiovasc Res 1981;15:164-70.

12. Jacob L, Boudaoud S, Pruna A, et al. Denervation does not prevent PEEP-induced antidiuresis and natriuresis in human kidney transplantation [Abstract]. Anesthesiology 1989;71:A189.

13. Robotham JL, Cherry D, Mitzner W, Rabson JL, Lixfeld W, Bromberger-Barnea B. A re-evaluation of the hemodynamic consequences of intermittent positive pressure ventilation. Crit Care Med 1983;11:783-93.

14. Robotham JL, Bell RC, Badke FR, Kinred MK. Left ventricular geometry during positive end-expiratory pressure in dogs. Crit Care Med 1985;13:617-24.

15. Dorinski PM, Hanlin RL, Gadek JE. Alterations in regional blood flow during positive end-expiratory pressure ventilation. Crit Care Med 1987;15:106-13.

16. Marini JJ, Culver BH, Butler J. Effect of positive end-expiratory pressure on canine ventricular function curve. $\mathbf{J}$ Appl Physiol 1981;51:1367-74.

17. Payen D, Lamer CH, Pilorget A, Moreau T, Beloucif S, Echter E. Evaluation of pulsed Doppler common carotid blood flow as a noninvasive method for brain death diagnosis. Anesthesiology 1990;72:222-9.

18. Brar HS, Platt LD. Reverse end-diastolic flow velocity on umbilical artery velocimetry in high-risk pregnancies: an 
ominous finding with adverse pregnancy outcome. Am J Obstet Gynaecol 1988;159:559-61.

19. Bredenberdg CE, Paskanik A, Fromm D. Portal hemodynamics in dogs during mechanical ventilation with positive end-expiratory pressure. Surgery 1981;90:817-22.

20. Bredenberdg CE, Paskanik AM. Relation of portal hemo- dynamics to cardiac output during mechanical ventilation with PEEP. Ann Surg 1983;198:218-22.

21. Winso O, Biber B, Gustavsson B, Milsom C, Niedman D. Portal blood flow in man during positive end-expiratory pressure ventilation. Intensive Care Med 1986;12:80-5.

\section{Help sought in identification of murder victim}

The Bureau of Criminal Investigation of the Department of State Police of the Commonwealth of Virginia is investigating the murder of an unidentified white male. They requested that THE JOURNAL OF THORACIC AND CARDIOVASCULAR SURGERY publish this announcement in the hopes that a cardiothoracic surgeon reading the JOURNAL may recognize the patient. If so, this physician should contact Dr. F. B. Presswalla at:

Commonwealth of Virginia

Department of Health

Office of the Chief Medical Examiner

$101 \mathrm{~A}$ Colley Avenue

Norfolk, VA 23507

Telephone number (804) 683-8366

Autopsy Number N-190-91

The victim was found on April 18, 1991, in a wooded area in Southampton County, Virginia, in the southeast part of Virginia. One year prior to his death, according to the pathologist, the victim underwent open heart surgery to repair a stab wound. The pathologist stated, "There is a median sternotomy surgical scar, 12 inches. There is a small scar (? old stab wound) in the right anterior mid-chest, just to the right of the midline." The pathologist wrote in reference to the heart in the autopsy report: "There is a sutured area in the right ventricle just to the right septum: cutdown into the area shows cartilage formation and fibrosis. This is an apparent heart injury that has been sutured (? old stab wound). No evidence of any coronary bypass or valvular sutures."

The descriptive data on the victim are as follows:

$\begin{array}{ll}\text { Name: } & \text { Unknown } \\ \text { Race: } & \text { White } \\ \text { Sex: } & \text { Male } \\ \text { Age: } & 25-35 \text { years } \\ \text { Height: } & 5^{\prime} 11^{\prime \prime} \\ \text { Weight: } & 247 \text { pounds } \\ \text { Eyes: } & \text { Brown } \\ \text { Hair: } & \text { Reddish brown } \\ \text { Scars: } & \text { Surgical scar on sternum }\end{array}$

